\title{
MANEJO INTEGRADO DE NEMATÓIDES NA CULTURA DA BANANEIRA
}

\author{
CECÍLIA HELENA SILVINO PRATA RITZINGER ${ }^{1} \&$ MARILENE FANCELLI
}

\begin{abstract}
RESUMO - O manejo integrado é uma exigência dos mercados importadores, sobretudo da Comunidade Européia (CE), rigorosa em requisitos de qualidade e sustentabilidade, que enfatiza primordialmente a proteção do meio ambiente, segurança alimentar, condições de trabalho, saúde humana e viabilidade econômica. Neste contexto, a utilização da matéria orgânica no manejo dos solos é uma das estratégias que mais benefícios traz à biodiversidade e conservação dos solos. É prática conhecida desde os primórdios da civilização. Contudo, sua utilização de forma efetiva depende ainda do conhecimento de muitas interações que ocorrem no agroecossistema. São apresentados alguns estudos de sua utilização no manejo integrado de fitonematóides e perspectivas de validação, com enfoque na cultura da bananeira e possibilidades de sua adoção para atender à demanda de sustentabilidade.
\end{abstract}

Termos para indexação: biodiversidade, agroecossistemas, sustentabilidade, pragas, controle biológico, mecanismos.

\section{INTEGRATED MANAGEMENT OF NEMATODES IN THE BANANA TREE CULTURE}

\begin{abstract}
The integrated management is a requirement of the European Community (CE) which demands rigorous quality product and sustainability, which is emphasized primarily on the environment protection, food safety, work conditions, human health and economical viability. In this context, the use of organic amendments for soil management is one of the strategies that brings the most benefits to soil biodiversity and conservation. This practice is known since the beginning of the civilization. Nevertheless, an effective use still depends on the knowledge of the many interactions in the agro-ecosystem. This review presents some studies of its use in integrated management of plant parasitic nematodes, validation perspectives focused in banana and use possibilities to assist the sustainability demand.
\end{abstract}

Index Terms: biodiversity, agro-ecosystems, sustainability, pest, biological control, mechanisms.

Os nematóides constituem o mais abundante grupo de animais multicelulares em número de indivíduos no universo, estimado em um milhão de espécies (Viglierchio, 1991). Muitas espécies são importantes na agricultura, pelos danos causados à produção, e outros, de vida livre, exatamente pelo seu efeito benéfico à agricultura. Os nematóides fitoparasitas, que promovem a destruição do sistema radicular, induzindo a formação de nodulações ou lesões necróticas nas raízes, impedem as plantas de absorverem água e nutrientes.

As perdas devidas ao ataque de nematóides na agricultura mundial estão estimadas em aproximadamente, US\$ 80 bilhões/ano (Agrios, 1997). Na agricultura americana, essas perdas são estimadas em US\$ 8 bilhões/ano, o que corresponde a $10 \%$ em relação à agricultura mundial (Barker et al., 1994). No Brasil, a quantificação de perdas não é precisa, devido principalmente às interações com danos provocados por pragas e outras doenças, condições climáticas, presença de plantas invasoras e inadequação de tratos culturais. Em vista do desconhecimento da importância econômica dos nematóides, esses organismos têm sido freqüentemente negligenciados nos agroecossistemas, somente assumindo "status" de patógeno quando sua população se encontra muito elevada, com prejuízos acentuados.

Na cultura da bananeira, as principais espécies fitoparasitas são Radopholus similis (Cobb, 1919) Goodey, 1933, diversas espécies de Meloidogyne spp., Helicotylenchus spp., Pratylenchus spp. e Rotylenchulus reniformis (Gowen \& Quénéhervé, 1990).

$\mathrm{O}$ controle químico tem sido o mais utilizado método de controle de nematóides na cultura da bananeira (Bridge, 2000). Porém, sabe-se que o uso indiscriminado de nematicidas, além de onerar a produção, coloca em risco a saúde dos aplicadores, consumidores e pode exercer forte pressão de seleção sobre os organismos presentes no solo, selecionando formas capazes de degradar o ambiente rapidamente. Uma das possíveis razões para o intenso uso de nematicidas concentra-se no fato de que as primeiras pesquisas com esses produtos em bananeira indicavam que o seu uso regular promovia um aumento na produção, desestimulando a realização de pesquisas sobre alternativas de controle.

Atualmente, as estratégias de manejo de fitonematóides prioritárias são aquelas que diminuem custos, aumentam a produção e não agridem o ambiente. A utilização de matéria orgânica, o controle biológico, o uso de variedades resistentes, a solarização, a rotação de culturas, o pousio, a inundação, o uso de cultivos intercalares e a cobertura do solo são abordados principalmente por reduzir a população dos nematóides e manter a biodiversidade nos diferentes agroecossistemas. Contudo, informações básicas são essenciais para o desenvolvimento dessas estratégias. Dentre elas, a identificação correta das espécies fitoparasitas, sua distribuição no solo, sua biologia, ecologia e interação parasita-hospedeiro, a qualidade do solo com base em suas características químicas e físicas, topografia e qualidade da água são extremamente importantes. A utilização de matéria orgânica, resíduos vegetais ou industriais, adubação verde, cobertura ou extratos vegetais com propriedades nematicidas, requer o mesmo rigor nas avaliações que o uso de pesticidas químicos (Zasada et al., 2002).

\section{Utilização de matéria orgânica}

Histórico da utilização da matéria orgânica para controle de fitonematóides

A utilização de matéria orgânica e de resíduos agrícolas ou subprodutos da agricultura é conhecida na China, Índia e civilização Inca desde os primórdios da humanidade. Contudo, os primeiros relatos científicos com base na experimentação sob ambientes controlados, em campo ou casa de vegetação, para controle de fitonematóides, foram conduzidos por Watson, nas décadas de 20 e 30, seguidos de vários outros trabalhos nas décadas seguintes (Watson, 1922; Holtz \& Vandecaveye, 1938; Linford et al., 1938; Lear, 1959). Todavia, essa prática isolada não é suficiente para o manejo de fitonematóides (Jacobsen, 1997; Cook, 2000; Bridge, 2000 e McSorley, 1998, 2001).

Recentemente, o interesse pela utilização da matéria orgânica no manejo de fitonematóides tem aumentado consideravelmente, não somente pela preocupação em reduzir a aplicação de produtos químicos nematicidas que causam grande impacto ambiental, mas também pela procura de alternativas que possam favorecer a sustentabilidade do agroecossistema (Mankau, 1968; Rodriguez-Kàbana, 1986; Brown, 1987; Stirling, 1991; McSorley \& Gallaher, 1993; Bridge, 2000).

Nos últimos cem anos, as práticas utilizadas para obter cultivos sadios têm se baseado na utilização de material de plantio sadio, isento de patógenos; utilização de área isenta de patógenos, com adequada fertilização; alta qualidade de água para irrigação e utilização de práticas de manejo protetoras e preventivas, como

\footnotetext{
${ }^{1}$ Embrapa Mandioca e Fruticultura Tropical, Caixa postal, 007, CEP 44380-000, Cruz das Almas, BA, e-mail: cecilia@ cnpmf.embrapa.br.
} 
utilização de variedades resistentes e pulverizações periódicas, impedindo o desenvolvimento de possíveis pragas (Cook, 2000). Nenhuma dessas táticas de manejo pode ser considerada revolucionária, mas pode-se questionar se são passíveis de adoção também pelo pequeno produtor de banana, bem como de atender à demanda de sustentabilidade (Bridge, 2000).

\section{Efeitos do manejo da matéria orgânica sobre nematóides}

No Brasil, diversos trabalhos vêm sendo desenvolvidos sob condições de casa de vegetação ou de campo, utilizando diversos tipos de resíduos orgânicos em cobertura, ou incorporados ao solo (Santos et al., 1981; Huang et al., 1981; Ponte \& Franco, 1983; Scramin et al., 1987; Aguillera et al., 1988; Silva et al., 1990). Os resultados revelam, na maioria das vezes, diminuição da população de nematóides. Em outros casos, registra-se maior produção, independentemente da população do fitonematóide. Essa visão simplista, via de regra, é importante razão para o descrédito de pesquisadores e produtores na utilização desse manejo para o controle de fitonematóides. Antes da tomada de decisões equivocadas, é necessária uma abordagem holística do agroecossistema, conhecer a dinâmica populacional e outras relações ecológicas, pois a ocorrência de fitonematóides na rizosfera não é um indicativo de perdas na cultura (Barker \& Koenning, 1998; Bridge, 2000). Ademais, no caso da bananeira, deve ser considerada a falta de conhecimento sobre a interação de nematóides com a broca-do-rizoma ou com a fusariose. Sabe-se que o ataque de um patógeno pode diminuir a resistência da planta, predispondo-a ao ataque por outras pragas (Bridge, 2000).

O mecanismo de ação da matéria orgânica na supressão de fitonematóides tem sido atribuído, na maioria das vezes, à melhoria da estrutura dos solos. Esta inclui desde mudanças no $\mathrm{pH}$, umidade e em propriedades químicas e físicas do solo, resultando em maior aeração, capacidade de retenção de água, melhoria na nutrição da planta ou no desenvolvimento de microrganismos que competem com os nematóides fitoparasitas, por meio da liberação de nutrientes à planta, aumento da população de predadores ou de microrganismos parasitas existentes no solo, ou por meio da liberação de metabólitos tóxicos devido à sua decomposição, como compostos fenólicos, $\mathrm{NH}_{3} \mathrm{ou}$ nitrito, íons de $\mathrm{Ca}^{+}$.

Contudo, essas mudanças são altamente dependentes da relação $\mathrm{C} / \mathrm{N}$ do material utilizado, do favorecimento ao crescimento e desenvolvimento de espécies antagônicas existentes no solo, dos metabólitos liberados por meio de sua de composição e da quantidade do material aplicado, seja para a supressão do fitoparasita, seja para a tolerância da planta. A espécie do fitonematóide presente, ou a condição da cultura, com relação à sua adaptabilidade e presença de tratos culturais adotados também devem ser considerados. Esses atributos podem ser importantes em situações específicas, pois a eficácia da matéria orgânica varia, dependendo da sua natureza, tipo de solo e espécie do fitonematóide. Além disso, a ação tóxica da decomposição da matéria orgânica por antagonistas, actinomicetos ou bactérias pode estar relacionada com a produção e liberação de quitinase. A produção de quitinase promove o rompimento da camada de proteção dos ovos dos fitonematóides, que é composta por quitina, resultando na eclosão prematura do estádio juvenil (Mian \& RodríguezKàbana., 1982; Stirling, 1991; Ritzinger \& McSorley, 1998 a; b; Rodríguez-Kàbana \& Kloepper, 1999).

Não obstante, a aplicação de matéria orgânica pode, por si só, promover benefício às plantas com relação à sua nutrição, bem como favorecer a manutenção de umidade, diminuindo o estresse hídrico. Dessa forma, a cultura poderá tolerar a presença dos fitoparasitas sem, contudo, apresentar queda acentuada da sua produção (McSorley \& Gallaher, 1995; Ritzinger et al., 1998; McSorley, 1998, Bridge, 2000).

Recente estudo sobre diferentes sistemas de produção no Recôncavo e Litoral Sul da Bahia tem revelado diferentes comportamentos nas populações de Helicotylenchus multicinctus (Blake, 1972) e Meloidogyne incognita Kofoid \& White (1919) Chitwood (1949) onde plantas sob melhor manejo apresentaram melhor desenvolvimento, mesmo sob aumento nas populações dos referidos nematóides (Santos et al., 2003).

\section{Matéria orgânica no manejo de nematóides em bananeira}

No Brasil, de acordo com Martins \& Farias (2002), a área ocupada com a agricultura orgânica está em torno de 100 mil hectares, com volume comercializado superior a US\$ 150 milhões, sendo $70 \%$ desses produtos destinados à exportação (Campanili, 2002). Sua expansão está associada, em grande parte, ao aumento de custos da agricultura convencional, à degradação do meio ambiente e à crescente exigência dos consumidores por produtos "limpos" ou livres de pesticidas (Darolt, 2004). Portanto, acredita-se que a utilização da matéria orgânica no manejo de fitonematóides e pragas possa vir a ser mais incentivada e estudada, principalmente na agricultura familiar.

O manejo orgânico baseia-se no uso de esterco animal, rotação e intercultivo com culturas rentáveis ou para cobertura do solo, adubação verde, compostagem, cultivares resistentes e mais adaptadas às condições da região. O controle biológico natural com o uso de agentes microbianos, de inseticidas e nematicidas botânicos, óleos, sabões, a liberação de inimigos naturais (predadores e parasitóides), armadilhas de feromônios e aplicação preventiva de fungicidas à base de enxofre, calda bordaleza e minerais tais como cobre e carbonato de cálcio, dentre outros, também são permitidos no manejo orgânico (Altieri, 1987).

Buscam-se, portanto, medidas voltadas para diminuir o uso de agrotóxicos na produção convencional, visando a otimizar o uso desses produtos ou manejos no sistema, adotando-se o princípio de atacar apenas os efeitos, e não, buscando o equilíbrio ecológico do sistema. Já os princípios agroecológicos, dentro dos quais a agricultura orgânica se insere, buscam aplicar o princípio da prevenção, fortalecendo o solo e as plantas por meio da promoção do equilíbrio ecológico em todo o ambiente de forma sustentável, ou seja, propiciando perpetuamente a colheita da biomasssa de um sistema, não comprometendo a sua capacidade de se renovar ou ser renovada (Gliessman, 2001; Martins \& Farias, 2002).

As quantidades de matéria orgânica utilizadas para promover a supressão dos fitoparasitas na cultura da bananeira são muito variáveis, pois, além da dependência das interações patógeno $\mathrm{x}$ hospedeiro e condições ambientais x cultura, dependem também do nível populacional da espécie e de sua correta identificação. É essencial que seja conhecido o mecanismo de supressão associado a cada material. A composição química dos ingredientes ativos, a concentração letal da matéria orgânica ou dos componentes para cada espécie de nematóide, o impacto deste material sobre as propriedades físicas e químicas do solo e a sua influência nos fatores bióticos do solo necessitam ser conhecidos. Desta forma, materiais orgânicos poderiam ser amplamente utilizados, principalmente em cobertura, pois, na maioria das vezes, os cultivos de bananeira estão localizados em áreas sujeitas à erosão e sujeitas à degradação, pois estão sob monocultivo por muitos anos consecutivos (Stirling, 1991; McSorley \& Gallaher, 1995; McSorley, 1998; Bridge, 2000; Zasada \& Ferris, 2003).

Trabalhos foram desenvolvidos sob condições de campo, em Petrolina-PE, com bananeira 'Prata-Anã', naturalmente infestadas por Meloidogyne incognita e M. javanica (Treub) Chitwood, 1949, com a aplicação de manipueira, urina de vaca, raspas e hastes de mandioca secas e trituradas, folhas e hastes de nim (Azadirachta indica Juss.) secas e trituradas, e combinações de urina de vaca e raspas de mandioca (Manihot esculenta Crantz), manipueira e nim, manipueira, nim e raspas de mandioca. Mesmo em condições adversas de estresse hídrico e chuvas prolongadas, a aplicação dos resíduos revelou redução da população final sob diferentes populações iniciais: alta (1.274 indivíduos $/ \mathrm{cm}^{3}$ de solo); média (329 indivíduos $/ \mathrm{cm}^{3} \mathrm{de}$ solo), 
e baixa (129 indivíduos $/ \mathrm{cm}^{3}$ de solo), quando comparados com os tratamentos-testemunha e tratamento químico utilizado.

Contudo, Bridge (2000) adverte para a necessidade de se estabelecerem níveis de dano e de se incrementarem estudos sobre a utilização de matéria orgânica e sua influência na atividade de microrganismos antagônicos e de outros microrganismos relacionados à decomposição de matéria orgânica, melhoria do crescimento da planta pelo maior e melhor desenvolvimento do sistema radicular, independentemente do aumento da população do nematóide.

\section{Controle biológico}

A existência de nematóides predadores, fungos-parasitas, bactérias e ácaros de solo pode exercer influência significativa na redução de fitonematóides (Stirling, 1991; University of California, 1991; Freitas et al., 1995). Esses inimigos naturais podem crescer em muitos tipos de solo, diferentemente de ano a ano, sendo freqüentemente encontrados associados ao sistema radicular. Geralmente, solos com alto teor de matéria orgânica possuem grande diversidade desses microrganismos naturais, enquanto nematicidas usados em pré-plantio podem eliminar a maioria desses microrganismos (University of California, 1991).

Diversos gêneros e espécies de fungos têm sido estudados no controle biológico de fitonematóides, entre os quais, Acremonium strictum Gams, Allomyces anomalus Emerson, Arthrobotrys arthrobotryoides (Berlese) Lindau, A. brochopaga (Drechsler) Schenck, Kendrick \& Pramer, Catenaria spp., Coniothyrium fuckelli Sacc., Cylindrocarpon destructans (Zinssmeister) Scholten, Dactylella candida (Nees) de Hoog \& van Oorschot, D. oviparasitica Stirling \& Mankau, Drechmeria coniospora (Drechsler) Gams \& Jansson, Exophiala pisciphilia McGinnis \& Ajello, Fusarium oxysporium Schlecht., F. solani (Mart.) Sacc., F. semitectum Berk. \& Rav., Gliocadium roseum Bain, Glomus fasiculatum (Thaxter) Gerdemann \& Trappe amend. Walker \& Koske, Harposporium spp., Hirsutella spp., Humicola grisea (van der Laan) Vinduska, Lagenidium caudatum Barron, Macrobiotophthora vermicola (McCulloch) Tucker, Monacrosporium spp., Nematoctonus spp., Paecilomyces lilacinus (Thom) Samson, P. nostocoides Dunn (Godoy et al., 1983) considerada espécie mutante de P. lilacinus, Periconia macrospinosa Lefebre \& A.G. Johnson, Rhopalomyces elegans Corda, Scytalidium fulvum Morgan-Jones \& Gintis, Stagonospora heteroderae Carris. Glawe \& Morgan-Jones, Verticillium spp., entre outros. Também são relacionadas diversas espécies de bactérias, como Clostridium butyricum Prazmowski, Desulfovibrio desulfuricans (Beijerinck) Kluyver \& van Niel, Pasteuria penetrans (Thorne) Sayre \& Starr, Pseudomonas spp. e Streptomyces anulatus (Beijerinck) Waksmann; bem como nematóides predadores, tais como Butlerius degrissei Grootaert \& Jaques, Iotonchus amphigonicus (Thorne) Andrassy, Iotonchus monhystera (Cobb) Jairajpuri, Labronema vulvapappillatum Meyl, Mononchus aquacticus Coetzee, Mylonchulus sigmaturus (Cobb) Altherr, Odontopharynx longicaudata de Man, Prionchus punctatus (Cobb) Andrassy. Alguns microartropodes como Alliphis halleri Canestrini, Caloglyphus berlesei Michael, Entomobyroides dissimilis Moniez, Gamasellodes vermivorax Walter, Hypoaspis aculeifer Canestrini, Lasioseius scapulatus Kennet, Sinella caeca Schott e Tyrophagus similis Volgin são relacionados no controle biológico de diversos fitonematóides (Stirling, 1991).

\section{Uso de variedades resistentes}

Dentre as estratégias de manejo, a utilização de resistência é, sem dúvida, uma das alternativas mais desejáveis considerando sua compatibilidade com outras práticas de manejo e não ser prejudicial ao ambiente (Fancelli, 2003).

A resistência da planta a pragas é geralmente definida pela habilidade da mesma em diminuir, inibir ou superar o ataque do patógeno (Wingard, 1953). Na Entomologia, a resistência é definida por características herdadas da planta que influenciam no dano causado pelo inseto-praga, sendo a não-preferência, a antibiose e a tolerância os três tipos de resistência. Freqüentemente, os nematologistas relacionam respostas do hospedeiro ao parasitismo dos nematóides com a habilidade da planta em suportar a reprodução do nematóide. A definição mais utilizada de resistência é a habilidade da planta em inibir a reprodução da espécie do nematóide. Portanto, uma planta suscetível pode ser intolerante, sendo supressa devido ao parasitismo do nematóide, ou pode ser tolerante com limitado crescimento, permitindo o desenvolvimento do nematóide. Esses diferentes conceitos em áreas distintas devem ser considerados para definir a resistência da planta a pragas e patógenos, os métodos utilizados para avaliar resistência e a natureza da interação da praga ou patógeno com o hospedeiro.

Trabalhos desenvolvidos por Costa (2004) revelam grande variabilidade patogênica de população de $R$. similis em genótipos de bananeira diplóides (AA) e triplóides (AAA) com diferentes graus de resistência. Independentemente do genótipo, a infecção causada pelas diferentes populações refletiu-se nas variáveis altura de plantas, peso da parte aérea e do sistema radicular. Uma relação positiva foi observada entre maiores números de nematóides por grama de raiz com efeito mais severos sobre a redução da altura de plantas e dos pesos das raízes quando comparados aos das plantas-controle.

Por meio de estudos histopatológicos das raízes infectadas por $R$. similis, a expansão das necroses entre as cultivares estudadas foi o indicador mais consistente do nível de resistência ao nematóide. As cultivares FHIA-18 e Thap Maeo mostraram-se mais resistentes à colonização por $R$. similis (Costa, 2004).

Considerando a variabilidade de isolados, a utilização de resistência não pode ser considerada a solução dos problemas causados por pragas, mas deve ter um papel importante no manejo em diversos sistemas de produção (Gonzáles-Rodriguez et al., 1997; Starr et al., 2002).

\section{Práticas culturais}

\section{Prática de adubação}

Práticas culturais que visam à diminuição do estresse nas plantas, como uma adequada adubação ou fertirrigação, rotação de culturas e pousio (com eliminação total das plantas infectadas e de plantas invasoras), também favorecem o manejo integrado de pragas e doenças, porque evitam a elevação da população dos patógenos por meio da manutenção do vigor das plantas. Práticas de adubação nitrogenada, fosfatada e potássica têm demonstrado respostas na redução da população de Meloidogyne, com menor número de galhas, bem como aumento no crescimento da planta. Por outro lado, a aplicação de cálcio tem sido menos efetiva.

\section{Inundação ou pousio}

A inundação da área infestada também contribui para a redução da população de fitonematóides. Contudo, muitas vezes, é difícil de ser adotada, pois depende da disponibilidade de água e do tipo de solo.

A eliminação de restos culturais e a utilização de rotação com culturas não-suscetíveis ou hospedeiras poderão contribuir sensivelmente para a redução da população de nematóides. Uma aração profunda do solo poderá trazer à superfície restos de raízes infectadas. Portanto, deixar o solo revolvido e exposto, efetuando essa operação periodicamente, poderá dessecar as raízes, dificultando o desenvolvimento dos fitonematóides. Essa prática, associada à eliminação de plantas invasoras hospedeiras por período de, no mínimo, seis meses e plantio de mudas sadias, pode reduzir 
sensivelmente a população de nematóides (Triverdi \& Barker, 1986; McSorley \& Dickson, 1995; McSorley, 2001). Entretanto, o uso da aração profunda exige cautela, pois poderá trazer graves problemas de conservação de solo.

A utilização de diversas práticas culturais combinadas com pousio e cultivos por dois anos consecutivos, utilizando-se de feijão (Phaseolus aureus Roxb.), sesbania (Sesbania sp.), cravo (Tagetes sp.), trigo e cevada mostrou ser efetiva na diminuição da população de Rotylenchulus reniformis Linford \& Oliveira, 1940 sem aumentar a população de Meloidogyne sp. e Tylenchorhynchus brassicae Siddiqi, 1961. Em outra pesquisa, o plantio de milheto (Pennisetum americanum CL) Leeke [P. Glaucum (L.) R. Br], 'Pearl Millet', em áreas com plantio de mamão, infestadas por Meloidogyne e Rotylenchulus, promoveu a redução da população desses nematóides. Entretanto, deve-se considerar a possibilidade de haver variação na eficiência de controle, pois pode haver diferenças no modo de ação entre cultivares da mesma espécie, Tagetes patula L. x T. ereta L. (McSorley, 1986; McSorley, 1992). Resultados se diferenciam na eficiência porque dependem também da quantidade ou do tipo de aplicação do material utilizado. Contudo, há necessidade de um monitoramento da população devido à possibilidade de as plantas invasoras suscetíveis elevarem a população dos fitonematóides (Borges et. al., 2003; Santos et al., 2003).

\section{Rotação de cultura}

A rotação de culturas pode afetar a sobrevivência de pragas e patógenos de plantas. A supressão é devida à quebra do ciclo desses organismos por um determinado tempo, o que dependerá basicamente das condições ambientais, além do nível de infestação e da espécie de praga ou patógeno considerada, bem como da presença de outros hospedeiros na área (Altieri, 1987; Johnson, et al., 2000; Ploeg, 2000; McSorley, 2001).

A utilização de plantas não-hospedeiras, que podem ser incorporadas ao solo ou servirem como cobertura, tem sua limitação por exigir um estabelecimento rápido e não permitir crescimento de plantas invasoras. Também podem constituir risco, se servirem como hospedeiros alternativos a outras pragas e doenças. Exige também critério econômico na escolha, principalmente se essa alternativa não oferecer retorno comercial ao agricultor. Por outro lado, a eficiência de matéria orgânica tem sido obtida sob altas dosagens de aplicação (Ritzinger \& McSorley, 1998 a; b, Ritzinger \& Souza, 2000).

\section{Cobertura morta ou cobertura verde}

Algumas espécies de gramíneas, leguminosas e compostas têm sido relatadas como sendo antagônicas a Meloidogyne e Rotylenchulus; todavia, o efeito nematicida tem sido relacionado à presença de substâncias que são liberadas por meio da decomposição dessas plantas que, por sua vez, são altamente dependentes da relação carbono/nitrogênio $(\mathrm{C} / \mathrm{N})$. A eficiência também pode ser afetada devido à flutuação que pode ocorrer na fertilidade do solo, teor de umidade e de matéria orgânica, e da densidade populacional dos nematóides. Assim sendo, a relação $\mathrm{C} / \mathrm{N}$ e a produção de massa fresca e seca da espécie, mudanças no $\mathrm{pH}$ do solo decorrentes do uso dessas espécies como cobertura, bem como o teor de matéria orgânica necessitam ser conhecidos para promover o manejo dos fitonematóides (Triverdi \& Barker, 1986; McSorley,1998; 2001; Ritzinger \& Alves, 2001).

Os nematóides podem ser influenciados de duas formas pela diversidade de espécies vegetais, ou seja, podem ser capturados ou mortos por certas plantas (ex: Tagetes erecta e T. patula x espécies de nematóides Pratylenchus, Tylenchorhynchus e Rotylenchulus) ou podem ser atraídos por cultura-armadilha (Altieri, 1987). Nesse último caso, as culturas-armadilha devem ser colhidas ou destruídas antes da eclosão dos nematóides, como é o caso do plantio de tomate juntamente com abacaxi (Ananas comosus L. (Merr.), em que a hortaliça é destruída antes que o nematóide das galhas possa produzir ovos.
Segundo Bridge (2000), embora a utilização de duas ou mais culturas possa propiciar redução na produtividade, a produção total da área cultivada é normalmente maior do que com o monocultivo. No oeste da África, é muito comum o cultivo de feijão, mandioca, milheto e vegetais entre linhas da bananeira. Todavia, o impacto desses diferentes cultivos nos diversos sistemas de produção sobre a dinâmica dos fitonematóides e perdas que possam causar, não têm sido estudados. Em multicultivos, uma determinada cultura pode ser arranjada numa seqüência de cultivos, ou em intercultivos, ou ainda por meio de uma combinação de seu cultivo no tempo e espaço. Em qualquer dessas técnicas, a população de pragas e patógenos pode ser diminuída ou aumentada, dependendo das espécies envolvidas. Para alguns nematóides, a escolha da cultura para rotação ou intercultivo é mais importante do que o período em que esse cultivo permanecerá no sistema. Em alguns casos, a rotação diminui sensivelmente a população do nematóide, mas quando na área é cultivado um hospedeiro suscetível, a população cresce rapidamente. Algumas vezes, os resultados obtidos também podem ser confundidos com os efeitos de espaçamento devido à diferente densidade de raízes. Por outro lado, alguns tipos de rotação podem ser efetivos para a redução da população de nematóides, mas, em outras situações, os resíduos deixados podem aumentar a população de insetos. Contudo, deve-se ater para o nível e infestação inicial (Pi), onde níveis altos, acima da densidade de equilíbrio, normalmente sofrem declínio da população após a prática de manejo. Para o manejo de nematóides, essa prática vem sendo utilizada com algum sucesso (McSorley, 2001). Em muitas situações, a rotação ou a cobertura vegetal é efetiva, todavia o custo de sua implantação, a aceitabilidade por parte do agricultor e a adaptabilidade da espécie é que determinam seu uso.

O sucesso da diversidade no sistema de cultivos para manejo de pragas e doenças requer conhecimentos detalhados sobre informações biológicas, incluindo a identificação da espécie, do isolado e da população presente; conhecimento do relacionamento entre a densidade e a produção, da existência e do número de hospedeiros e sobre a dinâmica da população. Infelizmente, muitas dessas informações são altamente específicas para cada localidade; contudo, onde não houver informações disponíveis, práticas e culturas alternativas devem ser testadas quanto à sua possível utilização. Em outras situações, o sucesso da alternativa de manejo não-químico depende do modo como uma informação especifica é obtida e utilizada (McSorley, 2001).

Resíduos deixados no solo podem aumentar a população de insetos, como no caso da broca-da-bananeira (Mesquita et al., 1983; Price, 1993). Entretanto, a cobertura do solo auxilia no controle de insetos por aumentar o número de inimigos naturais. Por exemplo, danos em pomares de maçã causados pela traça Cydia pomonella (Linnaeus, 1758) podem ser reduzidos por meio da utilização de cobertura com espécies vegetais que forneçam alimento para inimigos naturais da traça (Gliessman, 2001). Em bananeira, a manutenção da vegetação nativa nas entrelinhas das culturas serve de abrigo para inimigos naturais, como Hololepta quadridentata (Fabricius) inimigo natural da broca-do-rizoma-(Cosmopolites sordidus) da-bananeira (Martinez \& Godoy, 1991). Embora de eficiência discutível (Ostmark, 1974), os insetos predadores podem contribuir para prolongar a fase produtiva da cultura, integrando-se o uso de variedades resistentes, controle cultural, captura em iscas e melhoria no manejo da plantação (Koppenhöffer, 1993). Contudo, apesar do efeito favorável da cobertura morta sobre a população de $C$. sordidus, a eficiência dessa captura é variável de acordo com as condições climáticas e tipo de cobertura (Price, 1993). Como a infestação é avaliada com base no dano no rizoma e não no número de insetos nas iscas, algumas vezes, os resultados experimentais não são significativos.

\section{Perspectivas e validação de alternativas}

\section{Uso da matéria orgânica}


A utilização de matéria orgânica ou resíduos orgânicos em cobertura ou incorporada ao solo, a adubação e irrigação equilibradas, a utilização de plantas antagônicas, como Crotalaria spectabilis Roth e Crotalaria paulinea Schrank, podem reduzir, também, a população dos nematóides e favorecer a longevidade da cultura, por promover o desenvolvimento de microrganismos eficientes na redução dos fitoparasitas (Duncan, 1991; McSorley, 1992; Ritzinger et al., 1995; Ferraz \& Valle, 1997). Contudo, esses resíduos culturais podem, também, abrigar ou transmitir doenças e pragas a um cultivo subseqüente. Portanto, a época de incorporação de resíduos ao solo e sua compostagem longe da área de plantio são alternativas para contornar esses problemas e necessitam ser mais exploradas. Estudos econômicos e sobre a aplicabilidade dessa prática devem ser estimulados.

\section{Controle biológico}

A utilização do controle biológico pode seguramente reduzir a população de pragas e doenças e favorecer a longevidade da cultura. Porém, a eficiência deste tratamento está relacionada, entre outros fatores, principalmente ao nível populacional da praga, estado nutricional e idade do cultivo. Para viabilizar a utilização desses microrganismos no controle biológico, também são necessários estudos de multiplicação e distribuição desses microrganismos para sua disponibilização ao agricultor e, principalmente, obtenção de registros para comercialização.

A eficiência do controle microbiano depende de fatores bióticos, climáticos e não-climáticos. Dentre os fatores não-climáticos, o solo, apesar de ser o maior reservatório para os fungos entomopatogênicos, apresenta inúmeras limitações ao seu estabelecimento e atuação (Kermarrec et al., 1993), dentre os quais a composição mineral, orgânica e microbiana que podem influenciar na persistência, atividade biológica e comportamento epizootiológico. A variabilidade da população de nematóides no solo, muitas vezes, pode ser explicada pelas condições físicas do solo ou mesmo pelo histórico da cultura. Contudo, a distribuição e a multiplicação dos nematóides no solo nem sempre são uniformes e nem sempre alcançam nível de perda econômica em todas as situações. Portanto, o desafio do controle biológico com esses agentes deve ser melhor entendido dentro de cada sistema para sua sustentabilidade no manejo integrado (Barker \& Koening, 1998). A atividade empresarial na área de produção de inimigos naturais, apesar de ainda incipiente no Brasil, tende a aumentar para suprir as demandas atuais da agricultura.

Para diversas espécies de nematóides, são relatadas algumas plantas com propriedades nematicidas, entre as quais Azadirachta indica Juss., Chondrilla juncea L., Cucurbita pepo L., Dalbergia sissoo Roxb., Hannoa klaineana Planch., H. undulata (Guill. Perr.) Planch., Pinus radiata D. Don., Ricinus comunis L. e Shorea robusta Gaertn. (Stirling, 1991, Ritzinger \& McSorley, 1998 a; b).

\section{Solarização e biofumigação}

A solarização e a biofumigação são outras alternativas que podem ser incorporadas, haja vista os resíduos oriundos do bananal e de possíveis cultivos intercalares. Essa prática, associada a outros métodos, aumenta a eficiência do controle (Ghini, 1997). Neste enfoque, o efeito de gases produzidos durante a biodecomposição de matéria orgânica em cobertura e de restos culturais por meio da biofumigação surge como uma alternativa para o manejo integrado, pois, além de ter efeitos similares ao da fumigação convencional, a biofumigação melhora as características físicas, químicas e biológicas do solo (Bello et al., 2002; Casassa-Padrón et al., 2002). A eficiência da biofumigação é indicada pela propriedade do substrato orgânico utilizado, seja de natureza animal e vegetal, seja de industrial. Embora com características distintas, outras alternativas, como inundação, solarização e bioesterilização, podem ser consideradas complementares à biofumigação. A biofumigação é, portanto, uma alternativa de controle de patógenos baseada em recursos locais e reduz os impactos ambientais, além de promover a melhoria da qualidade da produção agrícola (Bello et al., 2002).

A eficácia da solarização para alguns patógenos de solo e plantas invasoras pode ser aumentada não somente pela espessura do plástico utilizado, mas também com a incorporação de alguns materiais, como esterco de galinha ou sulfato de amônio (McSorley \& McGovern, 2000). Contudo, devem ser aprofundados estudos sobre esses materiais a serem incorporados, porque, algumas vezes, a depender da dosagem utilizada, podem promover efeito fitotóxico.

\section{Resistência}

Relativamente, pouca atenção tem sido dada à incorporação de resistência a Musa contra nematóides, por causa das dificuldades de se trabalhar com um complexo genético e seu alto custo. Outros aspectos a serem considerados, relacionados a Pratylenchus spp. ou Radopholus sp., são a grande variabilidade no modo de reprodução e patogenicidade em suas populações; diferenças nas respostas dos hospedeiros à reprodução dos nematóides entre diversos experimentos, e a falta de informações relativas ao desenvolvimento radicular como resposta do hospedeiro à reprodução dos nematóides. A variabilidade na reprodução e também na patogenicidade de $R$. similis pode influenciar na interpretação de experimentos para resistência e tolerância (Bridge, 2000; Costa, 2003). Sugere-se, portanto, que cultivares promissoras oriundas de testes preliminares para avaliação da resistência/tolerância a diversas populações de Radopholus sp. e Pratylenchus spp. sejam testadas numa etapa final, utilizando-se de uma mistura de diferentes populações oriundas de diferentes regiões geográficas (De Waele \& Elsen, 2002).

Têm-se observado níveis de alta variabilidade de populações de fitonematóides nos acessos de bananeira, reforçando-se a necessidade de se conhecerem as espécies de fitonematóides e de se estabelecerem e determinar seus respectivos níveis de dano (Bridge, 2000; Costa, 2003). Além desses fatores que dificultam a estimativa da população de fitonematóides, a ocorrência da broca pode comprometer as avaliações para nematóides, seja em testes com produtos químicos, seja com plantas resistentes (Borges et al. 2003).

\section{Considerações finais}

A falta de resposta no aumento da produção após tratamento químico ou mesmo orgânico não necessariamente diz respeito à ineficiência no controle de determinada praga, ou que a mesma não causa danos à cultura. Outros fatores, como disponibilidade de água, fertilidade dos solos, graus-dia (GD), entre outros, contribuem para que a produção não aumente mesmo após a redução da população da praga. Dessa forma, a interpretação do manejo adequado deve ser feita com mais critério.

A adoção de práticas sustentáveis de controle de pragas tem sido lenta e bastante variável de acordo com a região e as culturas. Entre as estratégias, a rotação de culturas, o desenvolvimento de refúgios/abrigos para insetos benéficos e o uso de Bacillus thuringiensis Berliner têm sido mais evidentes. Todavia, a utilização de variedades resistentes, manejo da água, ajuste da data de plantio, controle físico e utilização de matéria orgânica necessitam ser revistas para a manutenção e a melhoria da biodiversidade dos agroecossistemas.

A maioria das pesquisas em banana e plátanos tem sido focalizada para Radopholus similis e Cosmopolites sordidus; contudo, há necessidade de se incrementarem pesquisas para se estabelecer nível de dano por agroecossistema. Os investimentos em insumos, como o controle biológico da broca-do-rizoma (principal praga da cultura), na certificação, no transporte e no trabalho dos técnicos, 
chegam a R\$50 mil por hectare (Campanili, 2002). Portanto, acredita-se que a utilização da matéria orgânica no manejo de fitonematóides e pragas possa vir a ser mais incentivada e estudada, principalmente na agricultura familiar.

Apesar do grande número de informações favoráveis aos sistemas de produção orgânica em relação aos convencionais (perdas de nitrogênio, riscos com pesticidas, biodiversidade), reconhece-se a necessidade de se desenvolverem métodos apropriados para avaliar a sustentabilidade dos sistemas agrícolas (Pacini et al., 2003).

Não existe resposta definitiva para o manejo de fitonematóides e pragas que atenda ao pequeno e grande produtor; contudo, práticas fitossanitárias, certificação da muda e monitoramento da população são de extrema importância para se estabelecer o manejo.

A utilização de intercultivos com a finalidade de quebrar o ciclo da praga e seu relacionamento com outras doenças têm se apresentado como mais eficiente do que a rotação de culturas não exploradas economicamente ou não comercializadas pelo produtor.

\section{AGRADECIMENTOS}

Ao Dr. John Bridge e Dr. Simon Gowen, Universidade da Inglaterra; Kenneth R. Barker, Universidade da Carolina do Norte; Dr. Robert McSorley, Universidade da Flórida e ao Dr. Gustavo Fallas, Costa Rica, pelo apoio no envio de informações atualizadas sobre manejo na cultura da bananeira.

\section{REFERÊNCIAS}

AGUILLERA, M.M.; VIEIRA, M.A.S.; MASUDA, Y. Aplicação de resíduos orgânicos para aumento de produtividade da cana-deaçúcar em solos infestados por nematóides. Nematologia Brasileira, Campinas, v.12, p.3-4, 1988.

ALTIERI, M.A. Agroecology: the scientific bases of alternative agriculture. Boulder: Westview press, 1987. 227p.

AGRIOS, G.N. Plant diseases caused by nematodes. In: GEORGE, N. AGRIOS, F.N. (Ed.). Plant Pathology. $4^{\text {th }}$ ed.. San Diego: Academic Press, 1997. p.565-597.

BARKER, K.R.; KOENNING, S.R. Developing sustainable systems for nematode management. Annual Review Phytopathology, Palo Alto, v.36, p.165-205, 1998.

BARKER, K.R.; HUSSEY, L.R.; KRUSBERG, L.R.; BIRD, G.W.; DUNN, R.A.; FERRIS, H.; FERRIS, V.R.; FRECKMAN, D.W.; GABRIEL, C.J.; GREWAL, A.E.; McGUIDWIN, A.E.; RIDDLE, D.L.; ROBERTS, P.A.; SCHIMITT, D.P. Plant and soil nematodes: societal impact and focus for the future. Journal of Nematology, Lakeland, v.26, p.127-137, 1994.

BELLO, A.; LÓPEZ-PÉREZ, A.A.; GARCÍA-ÁLVAREZ, A.; SANZ, R. Biofumigation and nematode control in the Mediterranean region. Nematology, Paris, v.4, p.143, 2002.

BORGES, A.L.; FANCELLI, M.; RITZINGER, C.H.S.P.; REINHARDT, D.H.; SILVA, M.N.B. da; TRINDADE, A.V.; SOUZA, L. da S. Aspectos gerais da produção orgânica de frutas. In: Alimentos orgânicos: produção, tecnologia e certificação. In: STRINGHETA, P. C.; MUNIZ, J. N. (Org.). Alimentos orgânicos: produção, tecnologia e certificação. Viçosa: UFV, 2003. p.235-288.

BRIDGE, J. Keynote: Nematodes of bananas and plantains in Africa: research trends and management strategies relating to the small scale farmer. Acta Horticulturae, Wageningen, n.540, p.391-408, 2000.

BROWN, R.H. Control strategies in low-value crops. In: BROWN, R.H.; KERRY, B.R. (Ed.). Principles and practice of nematode control in crops. Sydney: Academic Press, 1987. p.351-387.

CAMPANILI, M. Pequenos agricultores exportam banana orgânica. Disponível em: <http://www.estadao.com.br/ciencia/noticias/2002/ set/13/88.htm>. Acesso em: 20 mar. 2003.
CASASSA-PADRÓN, A.M.; PÉREZ-PÉREZ, E. MARÍN, M.; GONZÁLES, C.; CHIRINOS, D. SANDOVAL, L. Organic amendments as therapeutic treatment of guava trees (Psidium guajava L.) infested with Meloidogyne incognita in Zulia State, Venezuela. Nematology, Paris, v.4, p.145. 2002.

COOK, R.J. Advances on plant healthy management in the twentieth century. Annual Review Phytopathology, Palo Alto, v.38, p.95116, 2000.

COSTA, D. da C. Variabilidade patogênica e genética de Radopholus similis em genótipos de bananeira (Musa spp.) no Brasil. In: SIMPÓSIO BRASILEIRO SOBRE BANANICULTURA, 5., WORKSHOPDO GENOMAMUSA, 1., 2003. Paracatu. Anais... Cruz das Almas: Gráfica e Editora Nova Civilização, 2003. p.114-121.

COSTA, D. da C. Variabilidade patogênica e genética de Radopholus similis em bananeira no Brasil. 2004. Tese (Doutorado) Universidade de Brasília, Brasília. 2004.

DAROLT, M.K. Cenário internacional. Planeta Orgânico, Paraná, 2003. Disponível em <http//www.planetaorganico.com.Br>. Acesso em: 15 mar.2004.

DE WAELE, D.; ELSEN, A. Migratory endoparasites: Pratylenchus and Radopholus species. In: STARR, J.L.; COOK, BRIDGE, J. (Ed.). Plant resistance to parasitic nematodes. Wallingford: $\mathrm{CAB}$ International, 2002. p.175-206.

DUNCAN, L.W. Current options for nematode management. Annual Review of Phytopathology, Palo Alto, v.29, p.469-90, 1991.

FANCELLI, M. Resistência e alternativas de controle de pragas. In: SIMPÓSIO BRASILEIRO SOBRE BANANICULTURA, 5., WORKSHOP DO GENOMA MUSA, 1., 2003. Paracatu. Anais... Cruz das Almas: Gráfica e Editora Nova Civilização, 2003. p.127133.

FERRAZ, S.; VALLE, L.A.C. do. Controle de fitonematóides por plantas antagônicas. Viçosa: UFV, 1997. 73p. (Cadernos didáticos, 7).

FREITAS, L.G.; FERRAZ, S.; MUCHOVEJ, J.J. Effectiveness of different isolates of Paecilomyces lilacinus and an isolate of Cylindrocarpon destructans on the control of Meloidogyne javanica. Nematropica, Riverside, v.25, p.109-115, 1995.

GHINI, R. Desinfestação do solo com o uso de energia solar: solarização e coletor solar. Jaguariúna: Embrapa - CNPMA, 1997. 29p. (Circular, 1).

GLIESSMAN, S.R. Agroecologia: processos ecológicos em agricultura sustentável. 2.ed. Porto Alegre: Ed. Universidade/UFRGS, 2001. $653 p$.

GONZÁLES-RODRIGUEZ, J.B.; VENTURA-MARTÍN, J.DE LA C.; RODRIGUEZ-MORALES, S.; GALVEZ GUERRA, J.R.; JACOMINO, M. The behavior of clones of Musa spp. with regard to nematodes in Cuba. Infomusa, Montppellier, v.6, p.32-35, 1997.

GOWEN, S.; QUÉNÉHERVÉ, P. Nematode parasites of bananas, plantains and abaca. In: LUC, M.; SIKORA, R.A.; BRIDGE, J. (Ed.). Plant parasitic nematodes in subtropical and tropical agriculture. Wallingford: CAB, 1990. p.431-460.

HOLTZ, H.F.; VANDECAVEYE, S.C. Organic residues and nitrogen fertilizers in relation to the productivity and humus content of Palouse silt loam. Soil Science, Baltimore, v.45, p.143-163, 1938.

HUANG, C.S.; TENENTE, R.C.V.; SILVA, F.C.C.; LARA, J.A.R. Effect of Crotalaria spectabilis and two nematicides on numbers of Meloidogyne incognita and Helicotylenchus dihystera. Nematologica, Leiden, v.27, p.1-5, 1981.

JACOBSEN, B.J. Role of plant pathology in integrated pest management. Annual Review of Phytopathology, Palo Alto, v.35, p.373-391, 1997.

JOHNSON, A.W.; DOWLER, C.C.; HANDOO, Z.A. Population dynamics of Meloidogyne incognita, M. arenaria, and other nematodes and crop yields in rotation of cotton, peanut, and wheat under minimum tillage. Journal of Nematology, Lakeland, v.32, p.52-61, 2000. 
KERMARREC, A.; SIRJUSINGH, C.; MAULÉON, H; SARAH, J.L. Biological control of weevils and whitegrubs on bananas in the Caribbean: a review. In: GOLD, C.S.; GEMMIL, B. (Ed.). Biological and integrated control of highland banana and plantain pests and diseases. Cotonou: Proceedings of a Research Coordination Meeting, 1993. p.155-170.

KOPPENHÖFFER, A.M. Search and evaluation of natural enemies of the banana weevil. In: GOLD, C.S.; GEMMIL, B. (Ed.). Biological and integrated control of highland banana and plantain pests and diseases. Cotonou: Proceedings of a Research Coordination Meeting, 1993. p.87-96.

LEAR, B. Application of castor pomace and cropping of castor bean to soil to reduce nematode population. Plant Disease Reporter, St Paul, v.63, p.459-460, 1959

LINFORD, M.B., YAP, F.; OLIVEIRA, J.M. Reduction of soil populations of the root-knot nematode during decomposition of the organic matter. Soil Science, Baltimore, v.45, p.127-141, 1938.

MANKAU, R. Reduction of root-knot disease with organic amendment under semifield conditions. Plant Disease Reporter, St Paul, v.52, p.315-319, 1968.

MARTINEZ, N.B.; GODOY, F. Hololepta (Lioderma) quadridentata (Fabricius) depredador del gorgojo negro del plátano. In: REUNION DE LA ACORBAT, 9., 1989, Mérida. Memórias... Maracaibo: ACORBAT, 1991.p.363-369.

MARTINS, C.R.; FARIAS, R. de M. Produção orgânica de frutas Agropecuária Catarinense, Florianópolis, v.15, n.3, p.66-68, 2002.

McSORLEY, R. Nematode problems on banana and plantains in Florida Gainesville: IFAS, University of Florida, 1986. 4p. (Nematology Circular, 133).

McSORLEY, R. Alternative practices for managing plant-parasitic nematodes. American Journal of Alternative Agriculture, Oxfordshire, v.13, p.98-104, 1998.

MCSORLEY, R. Multiple cropping systems for nematode management: a review. Soil and Crop Science Society of Florida, Gainesville, v.60, p.132-142, 2001.

McSORLEY, R. Nematological problems in tropical and subtropical fruit tree crops. Nematropica, Riverside, v.22, p.103-116, 1992.

McSORLEY, R; DICKSON, D.W. Effect of tropical rotation crops on Meloidogyne incognita and other plant-parasitic nematodes. Journal of Nematology, Lakeland, v.27, p.535-544, 1995. supplement.

McSORLEY, R.; GALLAHER, R.N. Population dynamics of plantparasitic nematodes on cover crops of corn and sorghum. Journal of Nematology, Lakeland, v.25, p.446-453, 1993.

McSORLEY, R.; GALLAHER, R.N. Cultural practices improve crop tolerance to nematodes. Nematropica, Riverside, v.25, p.53-60, 1995.

McSORLEY, R.; McGOVERN, R.J. Effects of solarization and ammonium amendments on plant-parasitic nematodes. Journal of Nematology, Lakeland, v.32, p.537-541, 2000. supplement

MESQUITA, A.L.M.; CINTRA, F.L.D.; CALDAS, R.C. Evolução e danos do Cosmopolites sordidus em bananeira 'Prata' submetida a diferentes práticas de manejo. I. Primeiro seguidor. Cruz das Almas: EMBRAPA-CNPMF, 1983. 4p. (Pesquisa em Andamento, 15).

MIAN, I.H.; RODRIGUEZ-KABANA, R. Survey of the nematicidal properties of some organic materials available in Alabama as amendments to soil for control of Meloidogyne arenaria. Nematropica, Riverside, v.12, p.235-246, 1982.

OSTMARK, H.E. Economic insect pests of bananas. Annual Review of Entomology, Palo Alto, v.19, p.161-176, 1974.

PACINI, C.; WOSSINK, A.; GIESEN, G.; VAZZANA, C.; HUIRNE, R. Evaluation of sustainability of organic, integrated and conventional farming systems: a farm and field-scale analysis. Agriculture, Ecosystems \& Environment, Amsterdan, v.95, p.273$288,2003$.
PLOEG, A.T. Effects of amending soil with Tagetes patula cv. Single Gold on Meloidogyne incognita infestation of tomato. Nematology, Paris, v.2, p.489-493, 2000.

PONTE, J.J.; FRANCO, A. Efeitos nematicidas da manipueira em diferentes níveis de diluição. Fitopatologia Brasileira, Brasília, v.8, p.644, 1983

PRICE, N.S. Preliminary weevil trapping studies in Cameroon. In: GOLD, C.S.; GEMMIL, B. (Ed.). Biological and integrated control of highland banana and plantain pests and diseases. Cotonou: Proceedings of a Research Coordination Meeting, 1993. p.57-67.

RITZINGER, C.H.S.P.; ALVES, E.J. Nematóides. In: ALVES, E.J. (Ed.). Cultivo de bananeira tipo Terra. Cruz das Almas: Embrapa Mandioca e Fruticultura, 2001. p.117-122.

RITZINGER, C.H.S.P.; McSORLEY, R. Effect of castor and velvetbean organic amendments on Meloidogyne arenaria in greenhouse experiments. Journal of Nematology, Lakeland, v.30, p.624-631. 1998a. Supplement

RITZINGER, C.H.S.P.; McSORLEY, R. Effect of fresh and dry organic amendments on Meloidogyne arenaria in greenhouse experiments. Nematropica, Riverside, v.28, p.173-185, 1998b.

RITZINGER, C.H.S.P.; McSORLEY, R.; GALLAHER, R.N. Effect of Meloidogyne arenaria and mulch type on okra in microplot experiments. Journal of Nematology, Riverside, v.30, p.616-623, 1998. Supplement

RITZINGER, C.H.S.P.; SOUZA, J. da S. Situação dos problemas nematológicos na região norte do Brasil. In: CONGRESSO BRASILEIRO DE NEMATOLOGIA, 12., 2000. Anais... Uberlândia: Sociedade Brasileira de Nematologia, Instituto de Ciências Agrárias, Universidade Federal de Uberlândia, 2000. p.82-86.

RITZINGER, C.H.S.P.; VELASQUEZ-PEREIRA, J.; GALLAHER, R.N.; BUHR, K.L.; McSORLEY, R. Cotton disorders associated with plant nutrition status, soil fertility, and nematode occurrence. Gainsville: Agronomy Department, University of Florida, 1995. 28p. (Agronomy Research Report).

RODRIGUEZ-KABANA, R. Organic and inorganic nitrogen amendments to soil as nematode suppressants. Journal of Nematology, Lakeland, v.18, p.129-135, 1986.

RODRIGUEZ-KABANA, R.; KLOEPPER, J.K. Chitin-mediated changes in bacterial communities of the soil, rhizosphere and within roots of cotton in relation to nematode control. Soil Biology and Biochemistry, Oxford, v.31, p.551-560, 1999.

SANTOS, J.M.; FERRAZ, S.; OLIVEIRA, L.M. Efeito de fertilizantes nitrogenados na formação de galhas em raízes de cafeeiro atacada por Meloidogyne exigua e na eclosão de suas larvas. Fitopatologia Brasileira, Brasília, v.6, p.457-463, 1981

SANTOS, H.G. dos; RITZINGER, C.H.S.P.; LEDO, C.A. da S.; ALVES, E.J. Avaliação da população de fitonematóides em diferentes sistemas de produção de bananeira tipo terra em dois agroecossistemas. In: SIMPÓSIO BRASILEIRO SOBRE BANANICULTURA, 5., WORKSHOP DO GENOMA MUSA, 1., 2003. Paracatu. Anais... Cruz das Almas: Gráfica e Editora Nova Civilização, 2003. p.193-196.

SCRAMIN, S.; SILVA, H.P.; FERNANDES, L.M.S.; YAHN, C.A. Avaliação biológica de extratos de 14 espécies vegetais sobre Meloidogyne incognita raça 1. Nematologia Brasileira, Campinas, v.11, p.89-102, 1987.

SILVA, G.S.; FERRAZ, S.; SANTOS, J.M. Efeito de Crotalaria spp. sobre Meloidogyne javanica, $M$. incognita raça 3 e $M$. exigua. Fitopatologia Brasileira, Brasília, v.15, p.46-48, 1990.

STARR, J.L.; BRIDGE, J.;COOK, R. Resistance to plant-parasitic nematodes: history, current use and future potential. In: STARR, J.L.; COOK, BRIDGE, J. (Ed.). Plant resistance to parasitic nematodes. Wallingford: $C A B$ International, 2002. p.1-22.

STIRLING, G.R. Biological control of plant-parasitic nematodes. Wallingford: CAB International, 1991.282p. 
TRIVEDI, P.C.; BARKER, K.R. Management of nematodes by cultural methods. Nematropica, Riverside, v.16, p.213-236, 1986.

UNIVERSITY OF CALIFORNIA. Division of Agriculture and Natural Resources. Integrated pest management for Citrus. $2^{\text {nd }}$ ed. Oakland, CA, 1991.p.129-131. (Publication 3303).

VIGLIERCHIO, D.R. (Ed.). The World of Nematodes: a fascinating component of the animal kingdom. University of California: Davis, CA, 1991.266p.

WATSON, J.R. Bunch velvet bean to control root-knot. Gainesville: University of Florida Agricultural Experiment Station, 1922. (Bulletin, 163).
WINGARD, S.A. The nature of resistance to disease. In: THE YEAR book of Agriculture. Washington: Department of Agriculture, 1953. p.165-173.

ZASADA, I.; FERRIS, H. The complexity of implementing amendment based management systems for plant parasitic nematode suppression. Disponível em: <http://www.nematologists.org/ annualmeeting/abstract>. Acesso em: 09 out. 2003.

ZASADA, I.A.; FERRIS, H.; ZHENG, L. Plant sources of Chinese herbal remedies: Laboratory efficacy, suppression of Meloidogyne javanica in soil, and phytotoxicity assays. Journal of Nematology, Lakeland, v.34, p.124-129, 2002. 